\title{
Identification and characterization of a novel stress-responsive outer membrane protein Lip40 from Actinobacillus pleuropneumoniae
}

\author{
Xuehe $\mathrm{Hu}^{\dagger}$, Hao Yan ${ }^{\dagger}$, Ke Liu, Jiansheng Hu, Chao Qi, Jihong Yang, Yanli Liu, Jin Zhao and Jinlin Liu*
}

\begin{abstract}
Background: Actinobacillus pleuropneumoniae, a Gram-negative bacterium, is the causative agent of porcine pleuropneumonia, a highly contagious and often fatal disease. Because current vaccines confer limited protection against A. pleuropneumoniae infection, the development of more effective vaccines is urgently required. The identification of immunogenic and protective antigens, such as an outer-membrane lipoprotein, will advance this purpose.
\end{abstract}

Results: Sixty putative lipoproteins were predicted from the genomic sequence of A. pleuropneumoniae using multiple algorithms. Here, we focused on the characteristics of the putative lipoprotein Lip40 from A. pleuropneumoniae strain SLW01 (serovar 1). Lip40 shares sequence similarity with many bacterial lipoproteins, and the structural prediction of Lip40 suggests that it is similar to A. pleuropneumoniae TbpB. The N-terminus of Lip40 contains an interesting tandemly repeated sequence, Q(E/D/P)QPK. Real-time RT-PCR indicated that the expression of lip40 was significantly upregulated at $42^{\circ} \mathrm{C}$, at $16^{\circ} \mathrm{C}$, and under anaerobic conditions. Recombinant Lip40 (rLip40) produced in Escherichia coli BL21(DE3) was specifically recognized by porcine convalescent serum directed against $A$. pleuropneumoniae. Lip40 was confirmed to localize at the bacterial outer membrane, and its expression was significantly stimulated when $A$. pleuropneumoniae was cultured under various stress conditions. Lip40 also protected $75 \%$ of mice from fatal virulent A. pleuropneumoniae infection.

Conclusions: The immunogenic outer-membrane protein Lip40 is stress responsive, protects mice against infection, and might be a virulence determinant. Further investigation of Lip40 should expedite vaccine development and provide insight into the pathogenesis of A. pleuropneumoniae.

\section{Background}

Actinobacillus pleuropneumoniae is the etiological agent of porcine pleuropneumonia, a severe and often fatal respiratory disease of swine, which is associated with significant economic losses in industrialized pig production worldwide [1]. To date, 16 serovars of A. pleuropneumoniae have been identified [2]. The pathogenesis of $A$. pleuropneumoniae infection is associated with several virulence factors, including but not limited to exotoxins. Other factors, such as capsular polysaccharides, lipopolysaccharides, adhesins, proteases, outer-membrane

\footnotetext{
*Correspondence: liujinlin2009@126.com

${ }^{\dagger}$ Equal contributors

College of Life Sciences, Central China Normal University, Wuhan, Hubei 430079, China
} 430079, China

\section{() Biomed Central}

(c) 2015 Hu et al. Open Access This article is distributed under the terms of the Creative Commons Attribution 4.0 International License (http://creativecommons.org/licenses/by/4.0/), which permits unrestricted use, distribution, and reproduction in any medium, provided you give appropriate credit to the original author(s) and the source, provide a link to the Creative Commons license, and indicate if changes were made. The Creative Commons Public Domain Dedication waiver (http://creativecommons.org/publicdomain/zero/1.0/) applies to the data made available in this article, unless otherwise stated. proteins, and transcriptional regulators, are also reported to be involved in its pathogenesis [3-6].

Bacterial lipoproteins are a set of membrane-associated proteins characterized by a conserved lipid-modified cysteine, which play important roles in bacterial physiological processes [7]. The involvement of lipoproteins in the infection processes of many pathogens has received wide attention [8]. Lipoproteins play a key role in the bacterium's adhesion to the host cell, are involved in antibiotic resistance, and regulate the host immune response [8]. They have been reported to be pathogen-associated molecular patterns, which are recognized and captured by Toll-like receptors or other pattern-recognition receptors, inducing the activation of the immune cells and initiating the inflammatory processes [9]. Many immunogenic bacterial lipoproteins have been developed as candidate vaccines [8].

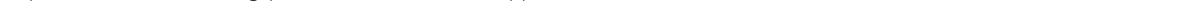


A putative lipoprotein, Lip40, containing the tandemly repeated sequence $\mathrm{Q}(\mathrm{E} / \mathrm{D} / \mathrm{P}) \mathrm{QPK}$ was observed among the 60 putative lipoproteins predicted from the published genomic sequence of $A$. pleuropneumoniae strain JL03. The lip40 gene from an A. pleuropneumoniae field isolate, SLW01 (serovar 1), was cloned and characterized, including its sequence features, predicted structure, immunogenicity, subcellular localization, expression after stimulation, and protective efficiency in mice, extending our understanding of this lipoprotein.

\section{Methods}

\section{Bacterial strains, plasmids, primers, and growth conditions}

The bacterial strains, plasmids, and primers used in this work are listed in Table 1. The primers used in this study were synthesized by Sangon Biotech (Shanghai, China). Escherichia coli strains were cultured in Luria-Bertani broth, supplemented with the appropriate antibiotic $(50 \mu \mathrm{g} / \mathrm{ml}$ ampicillin). Actinobacillus pleuropneumoniae strains were cultured in tryptic soy broth (TSB) or on tryptic soy agar (TSA) (Becton,
Dickinson \& Company, Franklin Lakes, New Jersey, USA), supplemented with $10 \mu \mathrm{g} / \mathrm{ml}$ nicotinamide adenine dinucleotide (Sigma-Aldrich Co., Ltd, St. Louis, Missouri, USA). For the anaerobic treatment, $A$. pleuropneumoniae was cultured under oxygen-free conditions ( $85 \% \mathrm{~N}_{2}, 10 \% \mathrm{H}_{2}$, and $5 \% \mathrm{CO}_{2}$ ) (Forma Anaerobic System, Thermo Fisher Scientific, Marietta, USA).

\section{Prediction of $A$. pleuropneumoniae lipoproteins}

The previously sequenced and annotated A. pleuropneumoniae strain JL03 was selected to identify the lipoproteins of A. pleuropneumoniae [10]. The programs used for lipoprotein prediction were: (i) ScanProsite (http:// prosite.expasy.org/) [11]; (ii) DOLOP (http://www.mrclmb.cam.ac.uk/genomes/dolop/) [12]; (iii) SignalP 4.1 (http://www.cbs.dtu.dk/services/SignalP/) [13]; (iv) PrediSi (http://www.predisi.de/) [14]; (v) Phobius (http:// phobius.binf.ku.dk/) [15]; and (vi) LipoP 1.0 (http:// www.cbs.dtu.dk/services/LipoP/) [16]. Possible lipoproteins were then subjected to a 'majority vote' selection procedure to exclude any false positive lipoproteins [17].

Table 1 Strains, plasmids, and primers used in this study

\begin{tabular}{|c|c|c|}
\hline Strain, plasmid and primer & Relevant characteristics & Source \\
\hline \multicolumn{3}{|l|}{ A. pleuropneumoniae } \\
\hline SLW01 & Serovar 1 & Weicheng Bei \\
\hline 13039 & Serovar 10 & Pat Blackall \\
\hline \multicolumn{3}{|l|}{ E. coli } \\
\hline $\mathrm{DH} 5 \mathrm{a}$ & Cloning vehicle: supE44 $\Delta$ lacU169 ( $\varphi 80$ lacZUM15) hsdR17recA1 endA1 gyrA96 thi-1 relA1 & Takara (Dalian, China) \\
\hline BL21(DE3) & $\begin{array}{l}\text { Expression host: } \mathrm{F}^{-} \text {ompT } \mathrm{r}_{\mathrm{B}}^{-} \mathrm{m}_{\mathrm{B}}^{-} \text {; DE3 is a } \lambda \text { derivative carrying lacl and T7 RNA polymerase genes } \\
\text { under placUV } 5 \text { control }\end{array}$ & Takara (Dalian, China) \\
\hline \multicolumn{3}{|l|}{ Plasmid } \\
\hline pMD18-T & E. coli cloning vector carrying an ampicillin resistance determinant & Takara (Dalian, China) \\
\hline pMD-lip40 & pMD18-T carrying lip40 gene of A. pleuropneumoniae strain SLW01. & This work \\
\hline pGEX-KG & N-terminal glutathione S-transferase (GST) fusion expression vector: pBR322 ori, Amp ${ }^{r}$ & {$[19]$} \\
\hline pGEX-lip40 & pGEX-KG carrying the lip40 gene for over-expression Lip40 protein & This work \\
\hline \multicolumn{3}{|c|}{ Primers (Primers were all synthesized in Sangon, Shanghai, China) } \\
\hline P1 & 5' ATG AAA AAC ATC ACA AAA TIT GCA G 3', upstream primer for lip40 gene cloning & This work \\
\hline P2 & 5' TTA CTT TTG TTG TाT TGC GCC AAA 3', downstream primer for lip40 gene cloning & This work \\
\hline P3 & 5' CGG TTC GAT TTG GTG TGT ATG A 3',upstream primer of lip40 gene for qRT-PCR analysis & This work \\
\hline P4 & 5' AAC AAG TAA GCA TCA CCT GTG T 3', downstream primer of lip40 gene for qRT-PCR analysis & This work \\
\hline P5 & $\begin{array}{l}\text { 5' AAG TGG CAG AGC TGG AAG AT 3', upstream primer of internal control gene rluC for qRT-PCR } \\
\text { analysis }\end{array}$ & This work \\
\hline P6 & $\begin{array}{l}\text { 5' TCA CAC CAA AAC TCA AGC CG 3', downstream primer of internal control gene rluC for qRT- } \\
\text { PCR analysis }\end{array}$ & This work \\
\hline P7 & $\begin{array}{l}\text { 5' TTG GAT CCT GTG GCA GTA AGA ACC ATT C 3', upstream primer with BamHl site (underlined) } \\
\text { comprising positions } 58 \text { to } 77 \text { of lip40 coding sequence }\end{array}$ & This work \\
\hline P8 & $\begin{array}{l}\text { 5' GGA AGC TाT TAC TाT TGT TGT TाT GCG C 3', downstream primer with Hindlll site (underlined) } \\
\text { comprising positions } 878 \text { to } 897 \text { of lip40 coding sequence }\end{array}$ & This work \\
\hline
\end{tabular}




\section{Cloning and bioinformatic analysis of Lip40 protein}

The genomic DNA was isolated from A. pleuropneumoniae SLW01 as described previously [18]. Specific primers for the lip40 gene were designed according to the conserved features of the lip40 sequence in the complete A. pleuropneumoniae genome. The lip40 gene was cloned by PCR, and the product was inserted into pMD18-T (Takara, Dalian, China), generating the plasmid pMD-lip40. The fragment inserted into pMD-lip40 was sequenced in both directions. The sequence features were analyzed with LipoP 1.0. A multiple sequence alignment was constructed with BioEdit (version 7.0). The structure of Lip40 was predicted by with the SWISS-model method (http://swissmodel.expasy.org/), and the Lip40 protein was superimposed onto the $\mathrm{N}$ lobe and $\mathrm{C}$ lobe of TbpB (Protein Data Bank [PDB]: 3HOL) with WinCoot.

\section{Reverse transcription-real-time quantitative PCR (RT- qPCR)}

Total RNA was extracted from an A. pleuropneumoniae culture with the RNeasy Mini kit (Qiagen, Shanghai, China), treated with DNase I (Invitrogen, CA, USA), and reverse-transcribed into cDNA with the Omniscript ${ }^{\circledR} \mathrm{RT}$ Kit (Qiagen), according to the manufacturer's protocol. The mRNA levels of $A$. pleuropneumoniae cultured under different conditions were measured with a SYBR Green-based method. The $r l u C$ gene was used as the internal control. qPCR was performed in a $20 \mu \mathrm{l}$ reaction volume containing $10 \mu \mathrm{l}$ of $2 \times$ SYBR Green Realtime PCR Master Mix (Toyobo, Osaka, Japan), $1 \mu \mathrm{l}$ of cDNA, $0.5 \mu \mathrm{l}$ each of the forward and reverse primers $(10 \mu \mathrm{mol} / \mathrm{l})$, and $8 \mu \mathrm{l}$ of $\mathrm{dH}_{2} \mathrm{O}$. An ABI 7500 Sequence Detection System was used for the amplification reactions, and qPCR was performed in triplicate. The $2^{-\Delta \Delta C t}$ method was used to calculate the relative expression of the lip40 gene.

\section{Expression and purification of Lip40 from E. coli}

The mature protein-coding sequence of Lip40 was cloned from the $A$. pleuropneumoniae genomic DNA using primers P7 and P8 (Table 1). The PCR products were then digested with $B a m \mathrm{HI} / H i n d I I I$ and ligated into the prokaryotic expression vector pGEX-KG [19], generating the recombinant plasmid pGEX-lip40. Recombinant Lip40 (rLip40) was then expressed from pGEX-lip40 in E. coli BL21(DE3). rLip40 was collected from the $E$. coli lysate and purified with a glutathione (GST)-Sepharose 4B column (Amersham Biosciences, Buckinghamshire, England). The immunoreactivity of the purified rLip40 was verified with western blotting, using porcine convalescent serum directed against $A$. pleuropneumoniae as the primary antibody.

\section{Production of polyclonal antibodies against rLip40}

Two female New Zealand white rabbits ( 2.5 kg; purchased from the Centre for Disease Control and Prevention [CDC] of Hubei Province, China) were used to produce polyclonal antibodies directed against rLip40. All the animal experiments in the present study were approved by the Animal Experiment Committee of Central China Normal University. Each rabbit was first injected intradermally with purified rLip40 $(650 \mu \mathrm{g})$. The purified protein $(0.5 \mathrm{ml})$ was emulsified with an equal volume of Freund's complete adjuvant (Sigma-Aldrich, USA). Animals were subsequently injected three times, at 15-day intervals, with the same immunogen emulsified with incomplete Freund's adjuvant with the same regimen. Antisera were collected 10 days after the third booster immunization.

The titers and specificity of the polyclonal antibodies were evaluated with a Lip40 enzyme-linked immunosorbent assay (ELISA). Briefly, flat-bottomed 96-well polystyrene ELISA plates (Haimen Shengbang, Haimen, China) were coated with $0.17 \mu \mathrm{g}$ of purified rLip40 diluted in $100 \mu \mathrm{l}$ of coating buffer (50 mM sodium carbonate, $\mathrm{pH}$ 9.6). The coated plates were washed three times with phosphate-buffered saline (PBS) plus $0.05 \%$ Tween 20 (PBST), blocked at $37{ }^{\circ} \mathrm{C}$ for $1 \mathrm{~h}$ with blocking buffer (5\% skimmed milk in PBST), and then washed three times with PBST. For the ELISA, $2 \mu \mathrm{l}$ samples and $198 \mu \mathrm{l}$ of PBST were added to each well in the first line of wells in the plate and $100 \mu \mathrm{l}$ of PBST was added to the rest of the wells. The samples were diluted and incubated at $37{ }^{\circ} \mathrm{C}$ for $40 \mathrm{~min}$. After four washes with PBST, $100 \mu \mathrm{l}$ of horseradish peroxidase (HRP)-conjugated secondary antibody (Southern Biotechnology Associates, Birmingham, USA), diluted 1:5000 in PBST, was added to each well, and the plates were incubated at $37^{\circ} \mathrm{C}$ for $30 \mathrm{~min}$. After five washes, $100 \mu \mathrm{l}$ of $3,3^{\prime}, 5,5^{\prime}$-tetramethylbenzidine color development solution (Biotime Biotech, Haimen, China) was added to each well. The plates were incubated at room temperature in the dark for approximately $10 \mathrm{~min}$, and the catalytic reaction was then stopped with $50 \mu \mathrm{l}$ of $1 \%$ SDS. The optical density was read at $630 \mathrm{~nm}\left(\mathrm{OD}_{630}\right)$ in an ELISA reader (PowerWave XS, Bio-Tek, Winooski, USA).

\section{Subcellular localization of Lip40}

The subcellular fractionation of A. pleuropneumoniae was performed as described previously [20]. In brief, 11 of bacterial culture was pelleted and resuspended in $10 \mathrm{ml}$ of solution A (0.2 M Tris- $\mathrm{HCl}[\mathrm{pH} 8.0], 1 \mathrm{M}$ sucrose, $1 \mathrm{mM}$ EDTA). Lysozyme was then added to the cell suspension to a final concentration of $1 \mathrm{mg} / \mathrm{ml}$, vortexed, and incubated at room temperature for $5 \mathrm{~min}$. $\mathrm{dH}_{2} \mathrm{O}(40 \mathrm{ml})$ was added to the swirling mixture before it was placed on ice. The cells were centrifuged at 
$200,000 \times g$ for $45 \mathrm{~min}$ at $4{ }^{\circ} \mathrm{C}$. The supernatant contained the periplasmic fraction. The pellet was resuspended in $7.5 \mathrm{ml}$ of ice-cold solution B (10 mM Tris- $\mathrm{HCl}[\mathrm{pH} 7.5]$, $5 \mathrm{mM}$ EDTA, $0.2 \mathrm{mM}$ DTT), supplemented with $50 \mu \mathrm{l}$ of DNase $(1 \mathrm{mg} / \mathrm{ml})$. The cells were broken by two passes at $10^{8} \mathrm{~Pa}$. The unbroken cells were pelleted by centrifugation at $4000 \times g$ for $10 \mathrm{~min}$ at $4{ }^{\circ} \mathrm{C}$. The supernatant was then centrifuged at $280,000 \times g$ for $4 \mathrm{~h}$ at $4{ }^{\circ} \mathrm{C}$. The supernatant contained the cytoplasmic fraction and the pellet contained the crude membranes, which were collected separately. The crude membrane pellet was resuspended in $9 \mathrm{ml}$ of solution C (50 mM Tris- $\mathrm{HCl}[\mathrm{pH} 8.0], 2 \%[v / v]$ Triton $\mathrm{X}-100,10 \mathrm{mM} \mathrm{MgCl}_{2}$ ), and centrifuged at $85,000 \times g$ for $30 \mathrm{~min}$ at $4{ }^{\circ} \mathrm{C}$. The supernatant contained the cytoplasmic membrane fraction. The pellet, containing the outer membrane, was washed in $1 \mathrm{ml}$ of solution $\mathrm{C}$, centrifuged at $85,000 \times g$ for $20 \mathrm{~min}$ at $4{ }^{\circ} \mathrm{C}$, washed three times with $500 \mu \mathrm{l}$ of $\mathrm{dH}_{2} \mathrm{O}$, and stored at $-20{ }^{\circ} \mathrm{C}$. The extracellular proteins were precipitated from the filtered culture supernatant with trichloroacetic acid at $4{ }^{\circ} \mathrm{C}$ overnight, followed by centrifugation $\left(17,700 \times g, 1 \mathrm{~h}, 4{ }^{\circ} \mathrm{C}\right)$, and were washed six times with $1 \mathrm{ml}$ of $96 \%$ ethanol. The pellet was dried and redissolved in $400 \mu \mathrm{l}$ of solution D (7 M urea, $2 \mathrm{M}$ thiourea, $4 \%$ CHAPS $[w / v], 30 \mathrm{mM}$ Tris$\mathrm{HCl},[\mathrm{pH} 8.0])$, and stored at $-20{ }^{\circ} \mathrm{C}$. The subcellular localization of the Lip40 protein was determined with western blotting using rabbit hyperimmune anti-rLip40 serum (1:400) as the primary antibody.

\section{Expression of Lip40 protein under stress conditions}

Actinobacillus pleuropneumoniae, cultured in TSB under normal conditions (aerobically at $37^{\circ} \mathrm{C}$ ) for $3 \mathrm{~h}$, was divided into four equal parts and incubated under anaerobic condition, or at $42{ }^{\circ} \mathrm{C}, 16{ }^{\circ} \mathrm{C}, 37{ }^{\circ} \mathrm{C}$ for another $3 \mathrm{~h}$, respectively. The cells were harvested by centrifugation, and the outer-membrane fractions were extracted as described above. The concentration of all outer-membrane samples prepared under different culture conditions were determined with a BCA protein assay kit (GenStar BioSolutions, Beijing, China). Equal volumes of the samples $(66 \mu \mathrm{g})$ were separated with $12 \%$ sodium dodecyl sulfate polyacrylamide gel electrophoresis and transferred to a nitrocellulose membrane. Western blotting was performed with rabbit hyperimmune anti-rLip40 serum (1:400) as the primary antibody. The expression of Lip40 was determined in triplicate. The expression levels of Lip40 in the outer-membrane samples were determined according to the density of the bands, with the Quantity One software.

\section{Vaccination and challenge of mice}

The protective efficacy of rLip40 was tested in a mouse vaccination/challenge model. Forty-eight 6-week-old specific-pathogen-free BALB/c mice (CDC, Hubei
Province, China) were randomly allocated to four groups of 12 animals. ApxI toxin was obtained from the supernatant of A. pleuropneumoniae strain 13039 (serovar 10) cultures, as described previously [21], and inactivated with $0.4 \%$ formaldehyde. Group 1 was inoculated with PBS and used as the negative control; group 2 was vaccinated with $150 \mu \mathrm{g}$ of rLip40; group 3 was vaccinated with $150 \mu \mathrm{g}$ of inactivated ApxI; and group 4 was vaccinated with a commercial trivalent inactivated vaccine (containing serovars 1, 2, and 7; purchased from Wuhan Keqian Biotech, China). For groups 2 and 3, the antigens were emulsified separately with complete Freund's adjuvant for the first immunization, and with incomplete Freund's adjuvant for the booster immunization. Serum samples were collected before each immunization and before the mice were challenged, and assayed with the Lip40-ELISA, as described above, and an ApxI-ELISA, as described previously [22]. Each group was intraperitoneally challenged with $2.5 \times 10^{6}$ colony-forming units of log-phase A. pleuropneumoniae SLW01 14 days after the second vaccination. The numbers of surviving mice were recorded every day for 5 days after the challenge.

\section{Results}

\section{Sixty putative $A$. pleuropneumoniae lipoproteins}

With the 'majority vote' approach [17], 60 of the 89 identified putative lipoproteins from A. pleuropneumoniae JL03 were predicted as lipoproteins, including some previously reported lipoproteins, such as TbpB (gene locus APJL_0250) [23] and PalA (gene locus APJL_0317) [24], as well as several previously reported putative lipoproteins [25], indicating that our prediction method and results are reliable. The sequence characteristics of the 60 lipoproteins are listed in Additional file 1: Table S1. Putative lipoprotein Lip40, containing a special $\mathrm{N}$-terminal tandemly repeated sequence, was further investigated in the present study.

\section{Lip40 has a conserved lipoprotein domain}

The lip40 gene from A. pleuropneumoniae SLW01 was cloned and sequenced (GenBank accession number: KP119767). The Lip40 protein was predicted to have the typical characteristics of lipoproteins with the LipoP 1.0 program, and contains the recognition sequence for signal peptidase II (LVITA ${ }^{\downarrow}$ CGSKN). The lipid modification site of Lip 40 was predicted to be located at Cys20. A multiple sequence alignment of Lip 40 and lipoproteins from Aggregatibacter actinomycetemcomitans and Cardiobacterium hominis showed that Lip40 is homologous to those lipoproteins (similarity $>33 \%$; Additional file 2: Figure S1). It also displayed similarity with several transferrin-binding proteins (Tbps) from pathogenic bacteria (similarity > $34 \%$; Additional file 3: Figure S2). The multiple sequence alignment showed that Lip40 is homologous to both the 
$\mathrm{N}$ - and C-lobes of A. pleuropneumoniae TbpB (Fig. 1a). Structural prediction revealed that the Lip40 protein consists of an eight-stranded $\beta$ barrel accompanied by a domain made up of four $\beta$ strands (Fig. 1b). Superimposition of Lip40 onto the $\mathrm{C}$ lobe and $\mathrm{N}$ lobe of TbpB (PDB: $3 \mathrm{HOL}$ ) indicated that they have high structural similarity (Fig. 1c and d). We also noted that Lip40 contains a tandemly repeated sequence, $\mathrm{Q}(\mathrm{E} / \mathrm{D} / \mathrm{P}) \mathrm{QPK}$, at its $N$ terminus. Actinobacillus pleuropneumoniae strain SLW01 Lip40 has nine tandem repeats. The numbers of repeats in the Lip40 homologues from other A. pleuropneumoniae strains were also calculated, and according to the available genome sequences of these strains, the repeat numbers differ among the different strains (8-27) (Table 2).

\section{Transcription of lip40 is stimulated by stress}

The expression of the lip 40 gene was analyzed under in vitro growth conditions mimicking the environment during infection, including anaerobic conditions and high temperature $\left(42{ }^{\circ} \mathrm{C}\right)$. The expression of lip 40 at low temperature $\left(16{ }^{\circ} \mathrm{C}\right)$ was also evaluated. As shown in Fig. 2, the transcription of the lip40 gene was significantly upregulated under stress relative to its transcription under normal culture conditions $\left(37^{\circ} \mathrm{C}\right.$, aerobic), and heat shock most strongly increased lip40 expression (45-fold). These results indicate that lip40 is responsive to temperature and anaerobic stress, and might be involved in the bacterial infection process.

\section{rLip40 is immunoreactive}

To express the Lip40 protein, the lip40 gene without the signal peptide was cloned from $A$. pleuropneumoniae SLW01, restricted, and ligated into the prokaryotic expression plasmid pGEX-KG. Escherichia coli cells containing pGEX-lip40 were induced with isopropyl- $\beta$-Dthiogalactopyranoside. The recombinant Lip40 protein

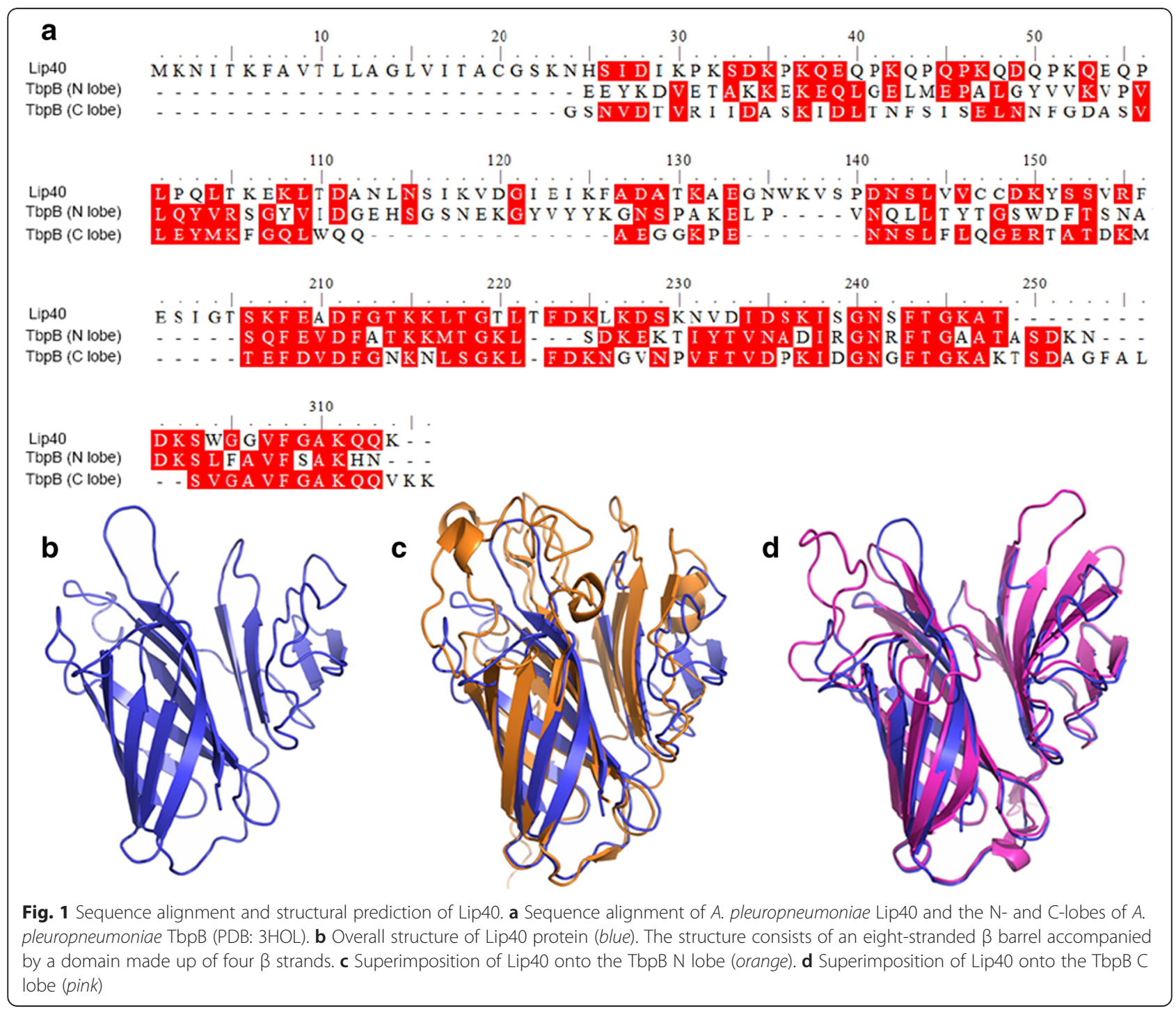


Table 2 Numbers of tandem repeats in different A. pleuropneumoniae strains

\begin{tabular}{lllllll}
\hline Strain & Serovar & Protein ID & $\begin{array}{l}\text { No. of total } \\
\text { amino acid }\end{array}$ & & & Tandem repeats \\
\cline { 6 - 7 } & & & No. of start position & No. of end position & No. of repeats \\
\hline SLW01 & 1 & AKJ32477 & 298 & 38 & 82 & 9 \\
4074 & 1 & ZP_00134150.1 & 383 & 38 & 167 & 26 \\
JL03 & 3 & YP_001652178.1 & 378 & 38 & 162 & 25 \\
M62 & 4 & ZP_07532361.1 & 372 & 52 & 156 & 21 \\
L20 & 5b & YP_001053852.1 & 293 & 38 & 77 & 23 \\
Femo & 6 & ZP_07336294.1 & 368 & 38 & 152 & 27 \\
AP76 & 7 & YP_001969011.1 & 388 & 37 & 172 & 26 \\
N273 & 13 & ZP_07545435.1 & 397 & 52 & 181 & 26 \\
\hline
\end{tabular}

was confirmed to be present in the soluble supernatant of the cell lysate with SDS-PAGE, as shown in Fig. 3a. The purification of rLip40 is shown in Fig. 3b. Western blotting revealed a band with a molecular weight of $\sim 56 \mathrm{kDa}$ for rLip40, whereas no signal was detectable for GST (Fig. 3c). This result confirms that porcine convalescent serum directed against $A$. pleuropneumoniae recognized and bound specifically to the rLip40 protein.

\section{Lip40 elicits a strong humoral immune response in rabbits}

Rabbits were immunized with four doses of purified rLip40 by intradermal injection. Serum samples were collected before each immunization and before euthanasia and the antibodies against rLip40 were tested with an ELISA, to verify the generation of a Lip40-specific immune response in rabbits. Lip40-specific antibodies appeared two weeks after the first immunization, and then increased quickly after the second and third injections (Additional file 4: Figure S3A). At the end of the immunization regimen, titration of the rabbit sera (at 55 days) showed the highest reactivity, with an

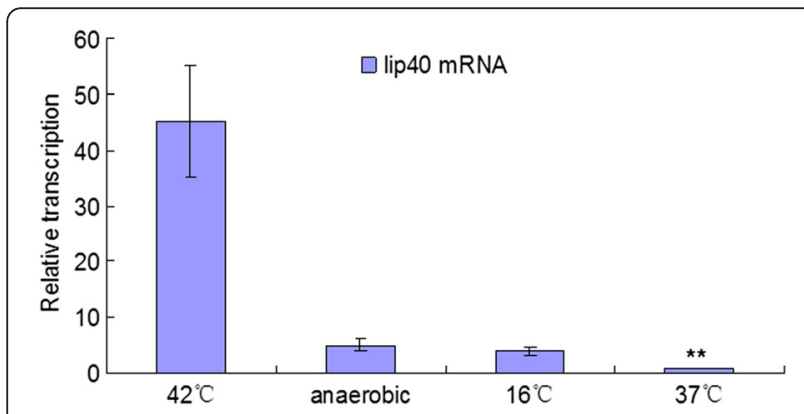

Fig. 2 Transcription changes in the A. pleuropneumoniae lip40 gene under different conditions. Relative expression of the lip40 gene during stimulation with $42{ }^{\circ} \mathrm{C}$, anaerobic conditions, or $16^{\circ} \mathrm{C}$ was normalized to that of lip40 expressed under normal culture conditions. The fold changes in lip40 expression are shown. Error bars indicate standard errors. Double asterisks indicate $P<0.01$, calculated with Student's $t$ test increase of up to $2 \times 10^{5}$-fold (Additional file 4: Figure S3B), suggesting that rLip40 stimulates a strong antibody response in rabbits. The same amount of purified GST was used to coat 96-well plates, and antibody directed against GST was evaluated with a GST-ELISA, as described for the Lip40-ELISA. The GST-specific IgG titer in the hyperimmune serum was only 1:3200 (Additional file 4: Figure S3B), much lower than that of the Lip40-specific antibody. Therefore, it appears that Lip40 played a major role in the elicitation of rabbit antibodies directed against the GST-Lip40 fusion protein.

\section{Lip40 is located in the outer membrane}

Although bioinformatic analysis showed that Lip40 is located on the outer membrane, experimental verification

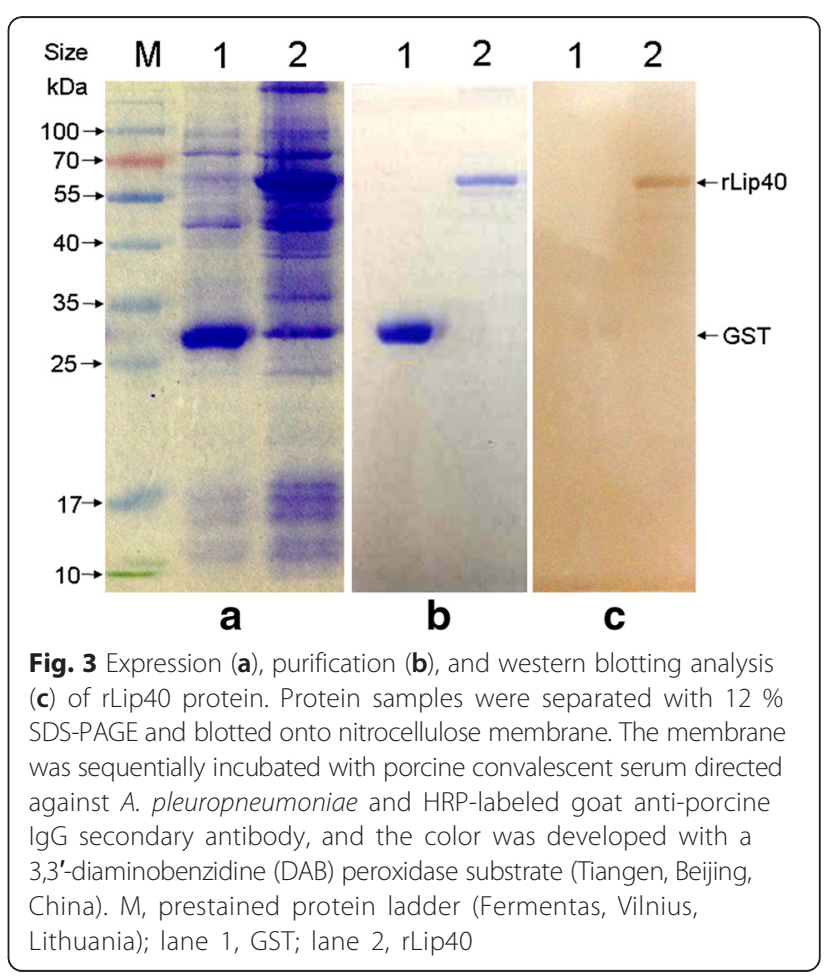


was required (data not shown). Subcellular fractions of A. pleuropneumoniae cells were extracted carefully, including the cytoplasmic proteins, periplasmic proteins, cytoplasmic membrane proteins, outer-membrane proteins (OM), and extracellular proteins (Fig. 4a). A band corresponding to Lip40 $(\sim 30 \mathrm{kDa})$ was observed in the outer-membrane fraction with western blotting, as shown in Fig. 4b. No Lip40 signal was observed in the other subcellular fractions. Our results confirm that Lip40 localizes to the outer bacterial membrane.

\section{Enhanced expression of Lip40 protein under stress}

To test whether the expression of Lip 40 protein in the outer-membrane fraction is stimulated by stress conditions, A. pleuropneumoniae cells cultured under anaerobic condition or high and low temperatures were collected, and their outer-membrane fractions were extracted and quantified. Equal amounts of samples $(66 \mu \mathrm{g})$ were separated by SDS-PAGE, and the expression levels were determined with western blotting (Fig. 5). In general, the expression of Lip40 protein was enhanced under stress conditions, especially at $42{ }^{\circ} \mathrm{C}$, and the Lip40 signal increased more than three-fold under heat shock. These results indicate that both the transcription of the lip40 gene and the translation of the Lip40 protein increased in response to stress. Therefore, Lip40 may be involved in the adaptation of A. pleuropneumoniae to environmental changes.

\section{Lip40 is protective in mice}

Serum samples from mice immunized with different antigens were analyzed with ELISAs (Fig. 6). All the mice were negative for rLip40 and ApxI before immunization. Serum samples from the mice inoculated with PBS (group 1) or ApxI (group 3) showed no detectable antibody against Lip40 during this study (Fig. 6a), whereas the sera from group 2, vaccinated with rLip40, were positive for Lip40 antibodies 14 days after the first vaccination, and the titer had increased by the second vaccination. The Lip40 antibody titers in group 2 were significantly higher than those in the other groups $(P<0.01$, Student's $t$ test; Fig. 6a). The mice in groups 1 and 2 were also free of ApxI antibodies, but the mice in group 3 showed a significantly stronger ApxI antibody response than the other groups $(P<0.01)$. As shown in Fig. 6b, group 4, vaccinated with commercial vaccine, also showed a strong ApxI antibody response after vaccination. The mice were challenged with virulent A. pleuropneumoniae SLW01 14 days after the second immunization, and the survival rates were recorded for 5 days after challenge (Fig. 7). No mice inoculated with PBS survived the challenge, and the protective efficacies of rLip40, ApxI, and commercial vaccine were $75 \%(9 / 12)$, $100 \%(12 / 12)$, and $91.7 \%$ (11/12), respectively. These results show that rLip40 protected more than half the animals $(75 \%)$ from the challenge. Thus, the protective efficacy of rLip40 was significantly higher than that of the negative control $(0 \%)$, indicating that rLip40 is immunoprotective and provides effective protection against $A$. pleuropneumoniae infection in mice.

\section{Discussion}

Lipoproteins are required for the virulence of some pathogenic bacteria. They play a variety of roles in host-

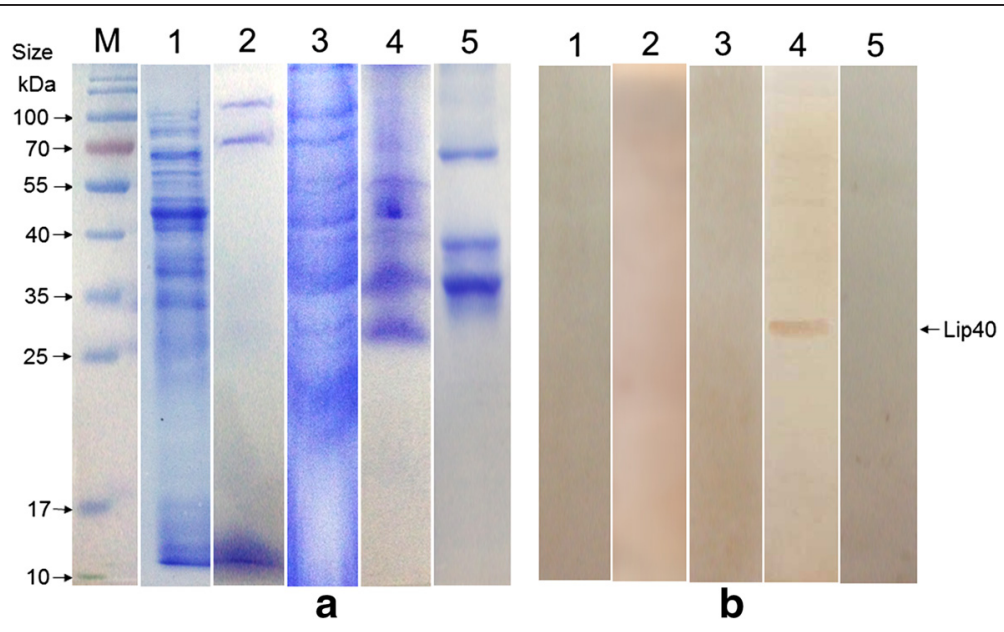

Fig. 4 Subcellular localization of Lip40. a SDS-PAGE analysis of A. pleuropneumoniae subcellular fractions. b Western blotting analysis. Subcellular fractions were separated with $12 \%$ SDS-PAGE, blotted onto nitrocellulose membranes, and incubated with rabbit anti-rLip40 polyclonal antibody. Color was developed with a DAB peroxidase substrate (Tiangen). M, prestained protein ladder; lane 1, cytoplasmic proteins (CP); lane 2, periplasmic proteins (PP); lane 3, cytoplasmic membrane proteins (CM); lane 4, outer-membrane proteins (OM); lane 5, extracellular proteins (EP). The nitrocellulose membranes displayed a band with a molecular weight of $30 \mathrm{kDa}$ in the outer-membrane fraction, indicating that Lip40 is located on the outer membrane. No signal was detected in the other subcellular fractions 

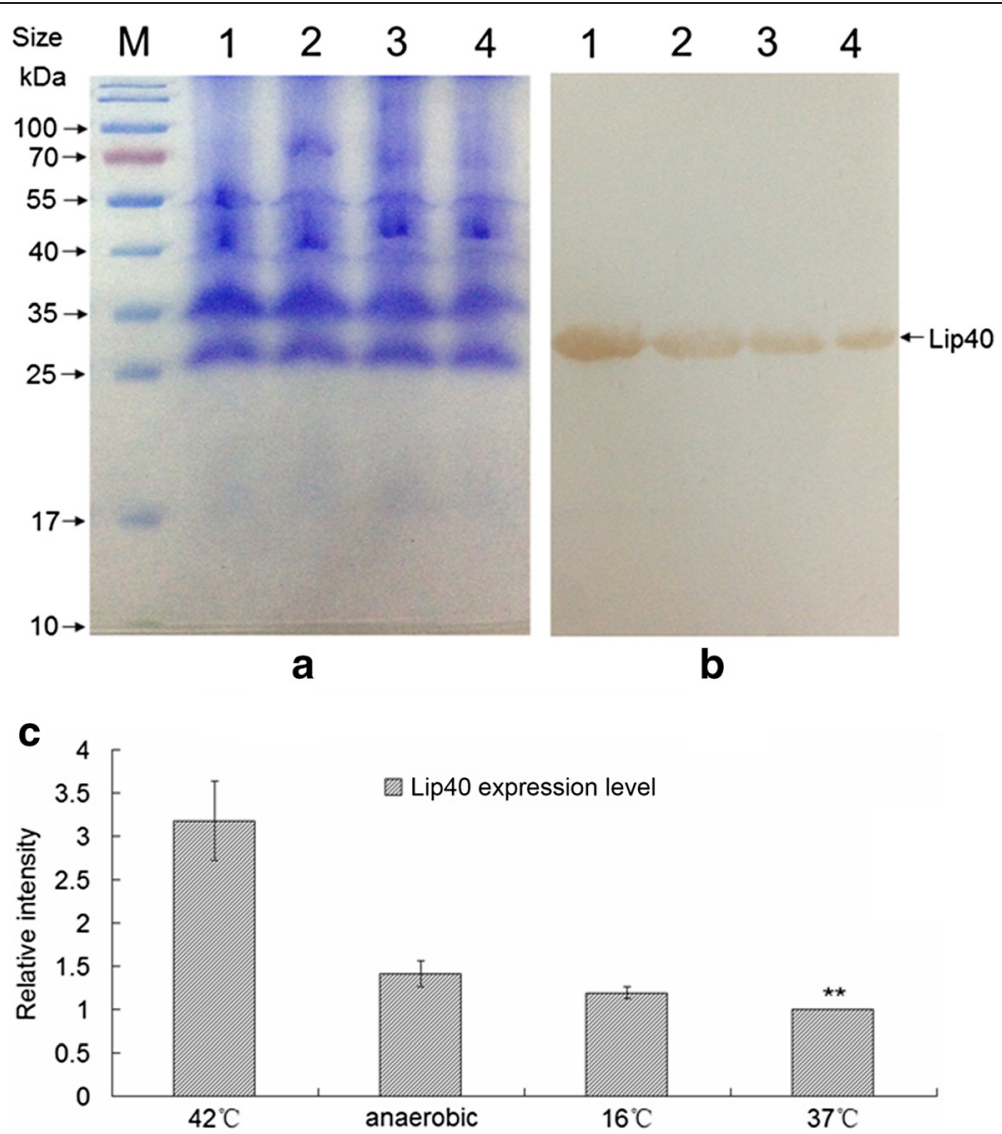

Fig. 5 Expression changes of Lip40 protein in the outer-membrane fraction. a SDS-PAGE. Actinobacillus pleuropneumoniae outer-membrane samples were extracted from cells after they were treated with heating, anaerobic conditions, or cold stimulation, or with normal conditions. Equal amounts $(66 \mu \mathrm{g})$ of outer-membrane proteins were analyzed with SDS-PAGE. $\mathbf{b}$ Western blotting analysis. The procedures were as described above. M, prestained protein ladder; lane 1, high temperature $\left(42^{\circ} \mathrm{C}\right)$; lane 2, anaerobic conditions; lane 3, low temperature $\left(16^{\circ} \mathrm{C}\right)$; lane 4 , normal conditions $\left(37^{\circ} \mathrm{C}\right)$. $\mathbf{c}$ Intensity of the bands was quantified with the Quantity One software. Relative expression levels were obtained by normalizing the intensity of stress-treated samples to that of untreated samples in each assay. Double asterisks $\left.{ }^{* *}\right)$ indicate a significant difference $(P<0.01)$ compared with the stress-treated samples (Student's $t$ test)

pathogen interactions, from surface adhesion and the initiation of inflammatory processes to the translocation of virulence factors into the host cytoplasm [8]. However, the pathogenesis of A. pleuropneumoniae lipoproteins has rarely been examined. Most such studies have focused on their immunogenicity and vaccine potential, including studies of TbpB [26-28] and OmlA $[29,30]$. These proteins were shown to be immunoprotective and important vaccine components (PleuroStar APP, Novartis Animal Health Inc., Switzerland). However, PalA was reported to inhibit the protective efficacy of ApxI and ApxII [24].

In the present study, a putative lipoprotein, Lip40, which contains an interesting tandemly repeated sequence, $\mathrm{Q}(\mathrm{E} / \mathrm{D} / \mathrm{P}) \mathrm{QPK}$, was characterized. Previous studies have demonstrated the relationship between coding tandem repeat (CTR) sequences and virulence in Chlamydia pecorum [31, 32]. CTRs at the incA locus encode a variable number of repeated motifs, and the number of repetitions in $i n c A$ is related to the virulence of C. pecorum strains [32]. Similarly, the number of tandem repeats in Lip40 varies within its homologues in different serovars of A. pleuropneumoniae strains. Therefore, the possible role of Lip40 in bacterial pathogenesis requires further characterization.

Oxygen deprivation and high temperature are two common stresses that $A$. pleuropneumoniae encounters during infection [5, 33]. Transcriptional profiles under anaerobic conditions [33] and acute disease [34] have been analyzed with microarray chips to discover potential virulence factors, which are usually considered to be upregulated under in vivo conditions or in a mimicked infection. This genome-wide transcriptional screening of the differentially expressed genes of A. pleuropneumoniae revealed no Lip40 homologue. In this study, we confirmed that Lip40 is upregulated at both the transcriptional and translational levels by stress. The mechanisms involved in the adaptive responses of $A$. 

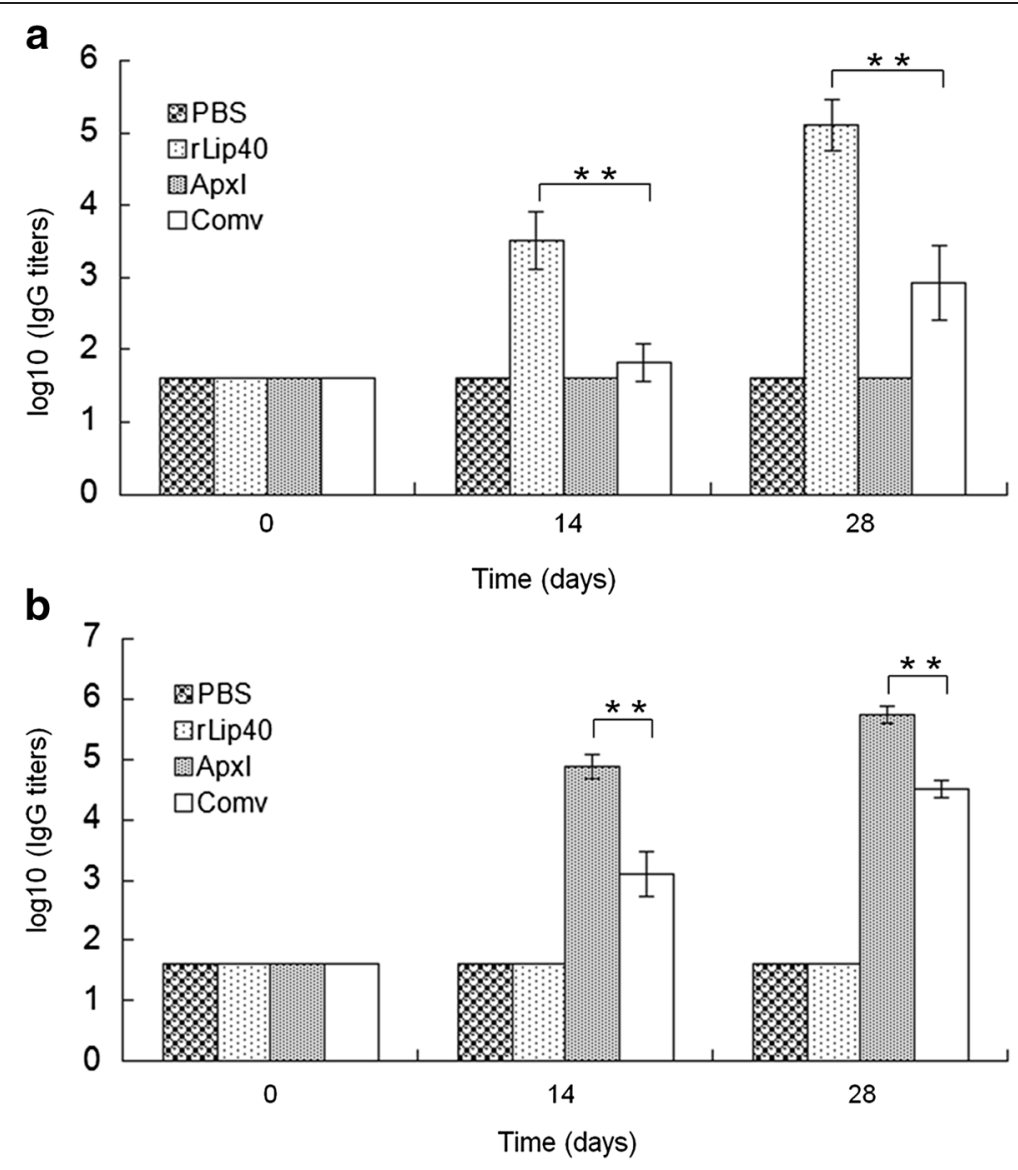

Fig. 6 Humoral responses in mice. Antibodies against rLip40 (a) and ApxI (b) were evaluated at different time points with ELISAs. ELISA titers are expressed as the logarithm $(\log 10)$ of the reciprocal of the highest dilution of serum with an $O_{630}$ value above that of the cutoff value for each mouse. Double asterisks ${ }^{* *}$ ) indicate $P<0.01$, calculated with Student's $t$ test

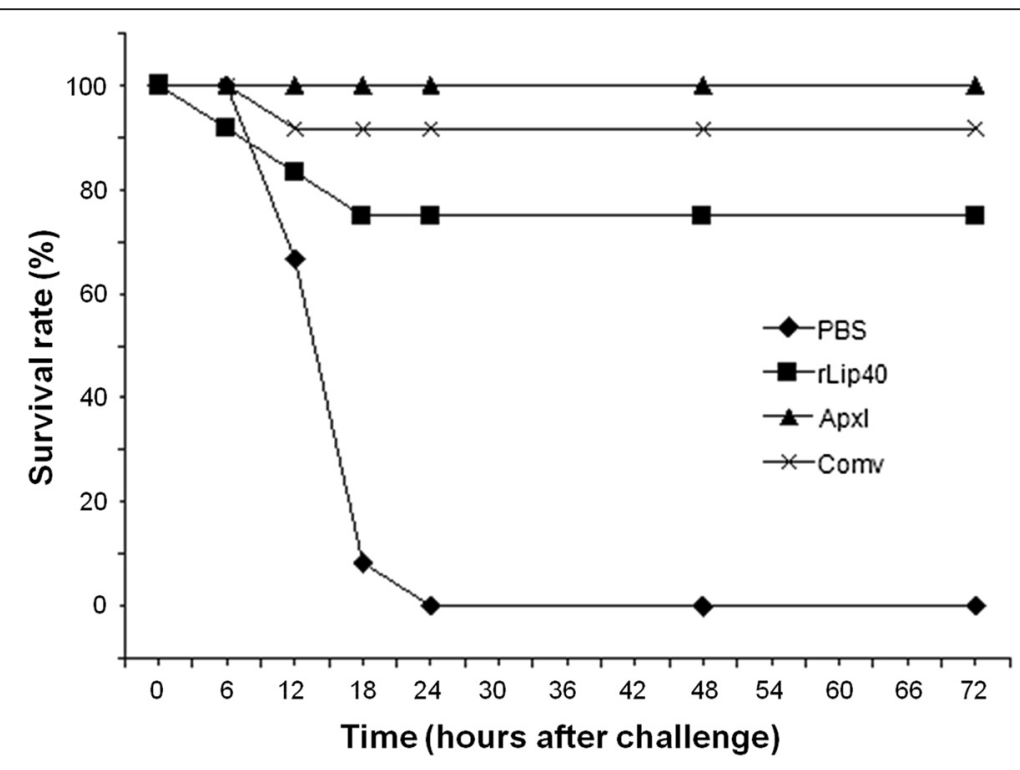

Fig. 7 Protection conferred by different antigens against A. pleuropneumoniae challenge in mice (survival rates). Numbers of surviving mice in each group was monitored for 5 days after challenge. Numbers of surviving mice did not change $72 \mathrm{~h}$ after infection 
pleuropneumoniae remain to be investigated. The effects of cold shock were tested as a typical environmental stress. Reports have suggested that the autumn-winter transition is one of the outbreak peaks of porcine pleuropneumonia in China [35], and the environmental temperature is always far lower than the normal growth temperature for mesophilic bacteria during this period. It is possible that the adaptation of A. pleuropneumoniae to cold stress is responsible for the infections observed at this time of year. Our results indicate that both the transcriptional and translational levels of Lip40 were elevated at $16{ }^{\circ} \mathrm{C}$ compared with those at $37^{\circ} \mathrm{C}$, suggesting that Lip40 is a factor in the responsiveness of $A$. pleuropneumoniae to low temperature. Therefore, we hypothesize that Lip40 is a potential virulence factor and involved in pathogen-environment and/or pathogenhost interactions.

Commercial vaccines, including whole-cell bacterins and second-generation subunit vaccines, only confer partial protection against $A$. pleuropneumoniae infection [29]. Therefore, it is essential that more effective vaccines be developed. The identification of conserved immunogenic antigens, especially outer-membrane-located proteins, will advance this purpose. Several outermembrane-associated proteins have been verified separately as vaccine components or candidate vaccines with various investigation methods, including $\mathrm{TbpB}, \mathrm{OmlA}$, OmpP2, OmpA, and OmpW [25, 36, 37]. In addition to their location on the outer bacterial membrane, many lipoproteins of Gram-negative bacteria are considered to be efficient candidate vaccines [38]. The putative outermembrane proteins and lipoproteins have been identified in the genome of Pasteurella multocida, a great step forward in the development of a protective vaccine against fowl cholera [39, 40]. Similar research was undertaken in A. pleuropneumoniae, and several lipoproteins were cloned and their protective efficacies were investigated [25]. Here, we report a putative lipoprotein, Lip40, confirmed to be an outer-membrane-localized stress-responsive factor. Purified rLip40 induced strong antibody responses when it was administered to rabbits. Mice vaccinated with rLip40 displayed a survival rate of $75 \%$, which was significantly higher than that of the negative control group ( $0 \%)$, indicating that rLip40 can be used as a component of vaccine against $A$. pleuropneumoniae infection. Our results provide additional confirmation of the importance of bacterial outer-membrane lipoproteins in vaccine development.

\section{Conclusions}

A putative lipoprotein, Lip40, from A. pleuropneumoniae was characterized and confirmed to be both immunogenic and localized to the outer membrane. Its expression is responsive to temperature and anaerobic stimuli.
Lip40 was also shown to be a potential protective antigen for vaccine development. Further investigation of the roles of Lip40 in A. pleuropneumoniae infection should provide insight into the pathogenesis of this bacterium.

\section{Additional files}

\begin{abstract}
Additional file 1: Table S1. Prediction of the potential lipoproteins (LPPS) of A. pleuropneumoniae (DOC $179 \mathrm{~kb}$ )

Additional file 2: Figure S1. Prevalence of A. pleuropneumoniae Lip40 homologues in other Gram-negative bacteria. Multiple sequence alignment was constructed with BioEdit (version 7.0). The conserved amino acid residues are shaded in red. Lipoproteins (LPPS) in Aggregatibacter actinomycetemcomitans (YP_004948053.1) and Cardiobacterium hominis (ZP_05705901.1) were used in the alignment analysis. (TIFF $2102 \mathrm{~kb}$ )

Additional file 3: Figure S2. Multiple sequence alignment. Homology between A. pleuropneumoniae Lip40 and Tbp proteins in Haemophilus sputorum (WP_007525245.1), Haemophilus pittmaniae (WP_007242241.1), and Neisseria sicca (WP_003766480.1) was analyzed with BioEdit (version 7.0). The conserved amino acid residues are shaded in red. (TIFF $5206 \mathrm{~kb}$ )

Additional file 4: Figure S3. Evaluation of polyclonal anti-Lip40 antibody. (A) Dynamics of the antibody responses elicited by rLip40. Serum samples were taken from rabbits before each immunization and 10 days after the last immunization. Serum samples were diluted by PBST $(1: 2000, \mathrm{v} / \mathrm{v})$ and evaluated by Lip40-ELISA. Results indicate the average $\mathrm{OD}_{630}$ values. (B) ELISA reactivity of anti-sera against rLip40 and GST. Anti-sera were collected 10 days after the last immunization, serially diluted and evaluated by Lip40-ELISA and GST-ELISA, separately. Results indicate the average $\mathrm{OD}_{630}$ values. (TIFF $2703 \mathrm{~kb}$ )
\end{abstract}

Competing interests

The authors declare that they have no competing interests.

\section{Authors' contributions}

$J L L$ conceived of the manuscript and designed all experiments, analyzed the data, and drafted the manuscript. XHH performed experiments, analyzed the data and drafted the manuscript. HY analyzed the data and drafted the manuscript. KL, JSH and YL analyzed the data. CQ, JHY and JZ provided instruments and experimental instructions. All authors read and approved the manuscript.

\section{Acknowledgements}

We would like to thank Dr. Weicheng Bei (at College of Veterinary Medicine, Huazhong Agricultural University, Wuhan, China) and Dr. Pat Blackall (at Queensland Alliance for Agriculture and Food Innovation, UQ, Australia) for the generous donation of A. pleuropneumoniae strains. We thank Dr. Jinrong Min (at Chromatin Structural Biology and Epigenetics, University of Toronto, Toronto, Canada) for the prediction of protein structure. This research was supported by grants from the National Nature Science Foundation of China (31101820) and the Fundamental Research Funds for the Central Universities (CCNU15A05037).

Received: 17 June 2015 Accepted: 29 August 2015

Published online: 25 November 2015

\section{References}

1. Fenwick B, Henry S. Porcine pleuropneumonia. J Am Vet Med Assoc. 1994;204:1334-40.

2. Foote SJ, Bossé JT, Bouevitch AB, Langford PR, Young NM, Nash JH. The complete genome sequence of Actinobacillus pleuropneumoniae L20 (serotype 5b). J Bacteriol. 2008;190:1495-6.

3. Chiers K, De Waele T, Pasmans F, Ducatelle R, Haesebrouck F. Virulence factors of Actinobacillus pleuropneumoniae involved in colonization, persistence and induction of lesions in its porcine host. Vet Res. 2010;41:65.

4. Li L, Xu Z, Zhou Y, Li T, Sun L, Chen H, et al. Analysis on Actinobacillus pleuropneumoniae LuXS regulated genes reveals pleiotropic roles of 
LuxS/AI-2 on biofilm formation, adhesion ability and iron metabolism. Microb Pathog. 2011;50:293-302.

5. Xie F, Zhang Y, Li G, Zhou L, Liu S, Wang C. The ClpP protease is required for the stress tolerance and biofilm formation in Actinobacillus pleuropneumoniae. PLoS One. 2013;8:e53600

6. Zhou Y, Li L, Chen Z, Yuan H, Chen H, Zhou R. Adhesion protein ApfA of Actinobacillus pleuropneumoniae is required for pathogenesis and is a potential target for vaccine development. Clin Vaccine Immunol. 2013;20:287-94.

7. Remans K, Vercammen K, Bodilis J, Cornelis P. Genome-wide analysis and literature-based survey of lipoproteins in Pseudomonas aeruginosa. Microbiology. 2010;156:2597-607.

8. Kovacs-Simon A, Titball RW, Michell SL. Lipoproteins of bacterial pathogens Infect Immun. 2011;79:548-61.

9. Brightbill HD, Libraty DH, Krutzik SR, Yang RB, Belisle JT, Bleharski JR, et al. Host defense mechanisms triggered by microbial lipoproteins through toll-like receptors. Science. 1999;285:732-6.

10. Xu Z, Zhou Y, Li L, Zhou R, Xiao S, Wan Y, et al. Genome biology of Actinobacillus pleuropneumoniae $\mathrm{JL} 03$, an isolate of serotype 3 prevalent in China. PLoS One. 2008:3:e1450.

11. Wu CH, Apweiler R, Bairoch A, Natale DA, Barker WC, Boeckmann B, et al. The Universal Protein Resource (UniProt): an expanding universe of protein information. Nucleic Acids Res. 2006:34:D187-91.

12. Babu MM, Priya ML, Selvan AT, Madera M, Gough J, Aravind L, et al. A database of bacterial lipoproteins (DOLOP) with functional assignments to predicted lipoproteins. J Bacteriol. 2006;188:2761-73.

13. Bendtsen JD, Nielsen H, von Heijne G, Brunak S. Improved prediction of signal peptides: SignalP 3.0. J Mol Biol. 2004;340:783-95.

14. Hiller K, Grote A, Scheer M, Münch R, Jahn D. PrediSi: prediction of signal peptides and their cleavage positions. Nucleic Acids Res. 2004;32:W375-9.

15. Käll L, Krogh A, Sonnhammer EL. A combined transmembrane topology and signal peptide prediction method. J Mol Biol. 2004;338:1027-36.

16. Juncker AS, Willenbrock $H$, Von Heijne G, Brunak S, Nielsen $H$, Krogh A. Prediction of lipoprotein signal peptides in Gram-negative bacteria. Protein Sci. 2003;12:1652-62.

17. Tjalsma H, van Dijl JM. Proteomics-based consensus prediction of protein retention in a bacterial membrane. Proteomics. 2005;5:4472-82.

18. Prideaux $C T$, Pierce L, Krywult J, Hodgson AL. Protection of mice against challenge with homologous and heterologous serovars of Actinobacillus pleuropneumoniae after live vaccination. Curr Microbiol. 1998;37:324-32.

19. Guan KL, Dixon JE. Eukaryotic proteins expressed in Escherichia coli: an improved thrombin cleavage and purification procedure of fusion proteins with glutathione S-transferase. Anal Biochem. 1991;192:262-7.

20. Thein M, Sauer G, Paramasivam N, Grin I, Linke D. Efficient subfractionation of gram-negative bacteria for proteomics studies. J Proteome Res. 2010;9:6135-47.

21. Devenish J, Rosendal S. Identification of the heat-labile hemolysin of Actinobacillus pleuropneumoniae serotype 1. Can J Vet Res. 1989;53:251-4.

22. Liu J, He Q, Chen H. Cloning, expression of apxl gene of Actinobacillus pleuropneumoniae and development of ELISA. Sci Agric Sin. 2003;5:578-82.

23. Tj K, Lee J. Cloning and expression of genes encoding transferrin-binding protein A and B from Actinobacillus pleuropneumoniae serotype 5. Protein Expr Purif. 2006:45:235-40.

24. van den Bosch $\mathrm{H}$, Frey J. Interference of outer membrane protein PalA with protective immunity against Actinobacillus pleuropneumoniae infections in vaccinated pigs. Vaccine. 2003;21:3601-7.

25. Chen X, Xu Z, Li L, Chen H, Zhou R. Identification of conserved surface proteins as novel antigenic vaccine candidates of Actinobacillus pleuropneumoniae. J Microbiol. 2012;50:978-86.

26. Baltes N, Hennig-Pauka I, Gerlach GF. Both transferrin binding proteins are virulence factors in Actinobacillus pleuropneumoniae serotype 7 infection. FEMS Microbiol Lett. 2002;209:283-7.

27. Goethe R, Gonzáles OF, Lindner T, Gerlach GF. A novel strategy for protective Actinobacillus pleuropneumoniae subunit vaccines: detergen extraction of cultures induced by iron restriction. Vaccine. 2000;19:966-75.

28. Gonzalez GC, Yu RH, Rosteck Jr PR, Schryvers AB. Sequence, genetic analysis, and expression of Actinobacillus pleuropneumoniae transferrin receptor genes. Microbiology. 1995;141:2405-16.

29. Chung JW, Küster-Schöck E, Gibbs BF, Jacques M, Coulton JW. Immunoproteomic analyses of outer membrane antigens of Actinobacillus pleuropneumoniae grown under iron-restricted conditions. Vet Microbiol. 2012;159:187-94.

30. Rossi CC, de Araújo EF, de Queiroz MV, Bazzolli DM. Characterization of the om/A gene from different serotypes of Actinobacillus pleuropneumoniae: a new insight into an old approach. Genet Mol Biol. 2013;36:243-51.

31. Yousef Mohamad K, Rekiki A, Myers G, Bavoil PM, Rodolakis A. Identification and characterisation of coding tandem repeat variants in incA gene of Chlamydophila pecorum. Vet Res. 2008;39:56

32. Mohamad KY, Kaltenboeck B, Rahman KS, Magnino S, Sachse K, Rodolakis A. Host adaptation of Chlamydia pecorum towards low virulence evident in co-evolution of the OMpA, incA, and ORF663 Loci. PLoS One. 2014;9:e103615.

33. Li L, Zhu J, Yang K, Xu Z, Liu Z, Zhou R. Changes in gene expression of Actinobacillus pleuropneumoniae in response to anaerobic stress reveal induction of central metabolism and biofilm formation. J Mcrobiol. 2014:52:473-81.

34. Klitgaard K, Friis C, Jensen TK, Angen $\varnothing$, Boye M. Transcriptional portrait of Actinobacillus pleuropneumoniae during acute disease - potential strategies for survival and persistence in the host. PLoS One. 2012;7:e35549.

35. Diao Y. Molecular epidemiological study on Actinobacillus pleuropneumoniae, Ph.D dissertation. Jinan, China: Library of Shandong Agricultural University; 2005. p. 17-8.

36. Buettner FF, Konze SA, Maas A, Gerlach GF. Proteomic and immunoproteomic characterization of a DIVA subunit vaccine against Actinobacillus pleuropneumoniae. Proteome Sci. 2011;9:23.

37. Oldfield NJ, Donovan EA, Worrall KE, Wooldridge KG, Langford PR, Rycroft $A N$, et al. Identification and characterization of novel antigenic vaccine candidates of Actinobacillus pleuropneumoniae. Vaccine. 2008;26:1942-54.

38. Ingolotti M, Kawalekar O, Shedlock DJ, Muthumani K, Weiner DB. DNA vaccines for targeting bacterial infections. Expert Rev Vaccines. 2010;9:747-63.

39. Al-Hasani K, Boyce J, McCarl VP, Bottomley S, Wilkie I, Adler B. Identification of novel immunogens in Pasteurella multocida. Microb Cell Fact. 2007;6:3.

40. E-komon T, Burchmore R, Herzyk P, Davies R. Predicting the outer membrane proteome of Pasteurella multocida based on consensus prediction enhanced by results integration and manual confirmation. BMC Bioinformatics. 2012;13:63.

\section{Submit your next manuscript to BioMed Central and take full advantage of:}

- Convenient online submission

- Thorough peer review

- No space constraints or color figure charges

- Immediate publication on acceptance

- Inclusion in PubMed, CAS, Scopus and Google Scholar

- Research which is freely available for redistribution 